\title{
Passivity analysis of neutral fuzzy system with linear fractional uncertainty
}

\author{
Jun Yang \\ School of Computer Science, Civil Aviation Flight University of China \\ Guanghan, Sichuan, 618307, China \\ Email: yj uestc@126.com \\ Wenpin Luo \\ College of Science, Sichuan University of Science and Engineering \\ Zigong, Sichuan, 643000, China \\ luowenp@126.com \\ Jinzhong Cui \\ School of Computer Science and Engineering, University of Electronic Science and Technology of China \\ Chengdu, Sichuan, 610054, China \\ Email: jzcui@uestc.edu.cn
}

\begin{abstract}
In this paper, the passivity analysis of TakagiSugeno (T-S) fuzzy neutral system with interval timevarying delay and linear fractional parametric uncertainty is investigated. Based on the Lyapunov- $\mathrm{K}$ rasovskii functional and the free weighting matrix method, delaydependent sufficient conditions for solvability of the passive problem are obtained in terms of Linear matrix inequalities (LMIS). Finally, a simulation example is provided to demonstr ate effectiveness and applicability of the theor etical results.
\end{abstract}

Index Terms-Passivity, Takagi-Sugeno fuzzy systems, Interval time-varying delay, Lyapunov-K rasovskii functional, L inear matrix inequalities (LMIs)

\section{INTRODUCTION}

The Takagi-Sugeno (T-S) model [1] has been paid considerable attention in the past two decades. It has been shown that the T-S model method gives an effective way to represent complex nonlinear systems by some simple local linear dynamic systems, and some analysis methods in the linear systems can be effectively extended to the TS fuzzy systems. Recently the T-S fuzzy neutral system has been introduced in [2] and the stability and stabilization analysis of fuzzy neutral systems have been extensively investigated, see, e.g.,[2-6] and the references therein.

On the other hand, The delay varying in an interval has strong application background, which commonly exists in many practical systems. For example, it has been described in [7] that the lower bound of the delay in the networked control systems is often larger than zero. The investigation for the systems with interval time-varying delay has been caused considerable attention, see [8-11] and the references therein.

The passivity theory, intimately related to circuit analysis methods, has received a lot of attention from the control community during the last several decades, see, e.g., [12,13]. It provides a nice tool for analyzing the stability of systems, and has found applications in diverse areas such as stability, complexity, signal processing. The fuzzy control systems associated with passivity have been studied preliminarily in [14]; [15] investigated the passivity and pacification of uncertain fuzzy systems; By utilizing the Lyapunov functional method, the Itô differential rule and the matrix analysis techniques, the passivity and pacification problems have been investigated for a class of uncertain stochastic fuzzy systems with time-varying delays [16].

However, to the best of the authors' knowledge, the passivity analysis of T-S fuzzy neutral system with interval time-varying delay and linear fractional parametric uncertainty has not been addressed, which motivates the present study.

Notations. $R^{n}$ and $R^{n \times m}$ denote, respectively, the $n$ dimensional Euclidean space and the set of all $n \times m$ real matrices. The notation $A>B$ means that $A-B$ is positive definite. $A^{\prime}$ represents the sum of $A$ and its transpose. I is the identity matrix with appropriate dimension. "*" denotes the elements below the main diagonal of a symmetric block matrix. $\mathrm{L}_{2}\left[\mathrm{t}_{0}, \infty\right)$ denotes the space of square integral functions on $\left[\mathrm{t}_{0}, \infty\right)$. 


\section{PROBLEM FORMULATION}

In this section, a class of neutral T-S fuzzy systems with interval time-varying delay and linear fractional parametric uncertainty is considered. For each $i \in S €$ $\{1,2, L, r\}$, where $r$ is the number of plant rules, the $i$ th rule of T-S fuzzy model is represented as follows:

Plant Rule i: IF $z_{1}(t)$ is $M_{i 1}, z_{2}(t)$ is $M_{i 2}, L, z_{p}(t)$ is $M_{\text {ip }}$, THEN

$$
\left\{\begin{aligned}
x(t)= & A_{i}(t) x(t)+B_{i}(t) x(t-\tau(t))+C_{i}(t) x(t-\tau(t)) \\
& +D_{i} w(t) \\
y(t)= & E_{i} x(t)+F_{i} x(t-\tau(t))+H_{i} w(t) \\
x(t)= & \psi(t), t \in\left[-\tau_{M}, 0\right]
\end{aligned}\right.
$$

where $z_{1}(t), z_{2}(t), L, z_{p}(t)$ are the premise variables, and each $M_{i 1}(l=1,2, L, p)$ is a fuzzy set; $x(t) \in R^{n}$ is the state variable, $y(t) \in R^{m}$ is the output vector, $w(t) \in R^{\prime}$ is the disturbance input belonged to $\mathrm{L}_{2}\left[\mathrm{t}_{0}, \infty\right) ; \psi(\mathrm{t})$ : $\left[-\tau_{M}, 0\right) \rightarrow R^{n}$ is a smooth vector-value initial function; $\tau(\mathrm{t}) \in\left[\tau_{\mathrm{m}}, \tau_{\mathrm{M}}\right]$ is the interval time-varying delay, where $0 \leq \tau_{\mathrm{m}} \leq \tau_{\mathrm{M}}$ and $\leq \mathrm{d} ; \mathrm{D}_{\mathrm{i}}, \mathrm{E}_{\mathrm{i}}, \mathrm{F}_{\mathrm{i}}, \mathrm{H}_{\mathrm{i}}$ are constant matrices with appropriate dimensions; $A_{i}(t), B_{i}(t)$, $C_{i}(t)$ are matrices with appropriate dimension and admissible linear fractional parametric uncertainties, that is, these matrices satisfy

$$
\begin{aligned}
{\left[A_{i}(t), B_{i}(t), C_{i}(t)\right] } & \\
& =\left[A_{i}, B_{i}, C_{i}\right]+L_{i} \Delta(t)\left[E_{1 i}, E_{2 i}, E_{3 i}\right], \\
\Delta(t) & =[l-F(t)]]^{-1} F(t), \\
I & -J J^{\top}>0,
\end{aligned}
$$

where $A_{i}, B_{i}, C_{i}, L_{i}, E_{1 i}(I=1,2,3)$ and $J$ are known real constant matrices with appropriate dimension, and

$F(t)$ is a matrix function satisfying

$$
\mathrm{F}(\mathrm{t}) \mathrm{F}^{\top}(\mathrm{t}) \leq \mathrm{I} \text {. }
$$

Remark 1. The uncertainty $\Delta(\mathrm{t})$ satisfying (3)-(5) is referred to as a linear fractional parametric uncertainty. Note that when $\mathrm{J}=0, \Delta(\mathrm{t})$ reduces to a norm-bounded parametric uncertainty that has been extensively investigated in the study of robust control problems.

Applying a center-average defuzzier, product inference and singleton fuzzifier, the dynamic fuzzy model in (1) can be represented by

$$
\left\{\begin{aligned}
& =\sum_{i=1}^{r} \mu_{i}(\mathrm{z}(\mathrm{t}))\left[\mathrm{A}_{\mathrm{i}}(\mathrm{t}) \mathrm{x}(\mathrm{t})+\mathrm{B}_{\mathrm{i}}(\mathrm{t}) \mathrm{x}(\mathrm{t}-\tau(\mathrm{t}))\right. \\
& \left.\left.+\mathrm{C}_{\mathrm{i}}(\mathrm{t}) \mathrm{t}-\tau(\mathrm{t})\right)+\mathrm{D}_{\mathrm{i}} \mathrm{w}(\mathrm{t})\right], \\
\mathrm{y}(\mathrm{t})= & \sum_{\mathrm{i}=1}^{\mathrm{r}} \mu_{\mathrm{i}}(\mathrm{z}(\mathrm{t}))\left[\mathrm{E}_{\mathrm{i}} \mathrm{x}(\mathrm{t})+\mathrm{F}_{\mathrm{i}} \mathrm{x}(\mathrm{t}-\tau(\mathrm{t}))+\mathrm{H}_{\mathrm{i}} \mathrm{w}(\mathrm{t})\right], \\
\mathrm{x}(\mathrm{t})= & \psi(\mathrm{t}), \mathrm{t} \in\left[-\tau_{\mathrm{M}}, 0\right],
\end{aligned}\right.
$$

where

$$
\mu_{\mathrm{i}}(\mathrm{z}(\mathrm{t}))=\frac{\prod_{\mid=1}^{\mathrm{p}} \mathrm{M}_{\mathrm{i} \mid}\left(\mathrm{z}_{\mathrm{l}}(\mathrm{t})\right)}{\sum_{\mathrm{i}=1}^{\mathrm{r}} \Pi_{\mathrm{l}=1}^{\mathrm{p}} \mathrm{M}_{\mathrm{i} \mid}\left(\mathrm{z}_{\mathrm{l}}(\mathrm{t})\right)}
$$

with $z(t)=\left(z_{1}(t), z_{2}(t), L, z_{p}(t)\right) ; M_{i l}\left(z_{1}(t)\right)$ is the grade of membership of $z_{l}(t)$ in $M_{i l}$; For notational simplicity, $\mu_{\mathrm{i}}$ is used to represent $\mu_{\mathrm{i}}(\mathrm{z}(\mathrm{t}))$ in this paper. By the definition in (7), it follows that $\mu_{\mathrm{i}} \geq 0$ and $\sum_{\mathrm{i}=1}^{\mathrm{r}} \mu_{\mathrm{i}}=1$.

Definition 1 [17]. The system (1) is called passive if there exists a scalar $\gamma>0$ such that

$$
-\gamma \int_{0}^{t_{\rho}} \mathrm{w}^{\top}(\mathrm{s}) \mathrm{w}(\mathrm{s}) \mathrm{ds} \leq 2 \int_{0}^{\mathrm{t}_{\rho}} \mathrm{w}^{\top}(\mathrm{s}) \mathrm{y}(\mathrm{s}) \mathrm{ds}, \mathrm{t}_{\rho} \geq 0
$$

for all solution of (1) under zero initial condition.

Lemma 1 [18]. Suppose $\Delta(\mathrm{t})$ is given by (3). Given matrices $M=M^{\top}, L$ and $E$ of appropriate dimension, the following statements are equivalent:

(i) the inequality

$$
\mathrm{M}+\mathrm{L} \Delta(\mathrm{t}) \mathrm{E}+\mathrm{E}^{\top} \Delta^{\top}(\mathrm{t}) \mathrm{L}^{\top}<0
$$

holds for all $\mathrm{F}(\mathrm{t})$ satisfying $\mathrm{F}(\mathrm{t}) \mathrm{F}^{\top}(\mathrm{t}) \leq \mathrm{l}$;

(ii) for $\varepsilon>0$

$$
\left[\begin{array}{ccc}
\mathrm{M} & \varepsilon \mathrm{E}^{\top} & \mathrm{L} \\
* & -\left.\varepsilon\right|^{\mathrm{l}} & \varepsilon \mathrm{J}^{\top} \\
* & * & -\varepsilon \mid
\end{array}\right]<0 .
$$

Lemma 2 [11]. Let $\Xi_{1}, \Xi_{2}$ and $\Omega$ be constant matrices appropriate dimensions and $0 \leq \tau_{\mathrm{m}} \leq \tau(\mathrm{t}) \leq \tau_{\mathrm{M}}$, then

$$
\left(\tau(\mathrm{t})-\tau_{\mathrm{m}}\right) \Xi_{1}+\left(\tau_{\mathrm{M}}-\tau(\mathrm{t})\right) \Xi_{2}+\Omega<0
$$

if and only if

$$
\left(\tau_{\mathrm{M}}-\tau_{\mathrm{m}}\right) \Xi_{1}+\Omega<0
$$

and

$$
\left(\tau_{\mathrm{M}}-\tau_{\mathrm{m}}\right) \Xi_{2}+\Omega<0
$$

hold. 


\section{MAIN RESULTS}

Theorem 1. For a prescribed $\gamma>0$, scalars $\tau_{\mathrm{m}}$ and $\tau_{\mathrm{M}}$, the system (1) is passive if there exist scalars $\varepsilon_{\mathrm{i}}(\mathrm{i} \in \mathrm{S})$, matrices $P>0, Z>0, Q_{1}(I=1,2,3), \quad S_{k}>0, S_{k}>0$, $M_{\mathrm{ki}}, \mathrm{N}_{\mathrm{ki}},(\mathrm{k}=1,2 ; \mathrm{i} \in \mathrm{S})$ such that the following LMIs hold

$$
\Xi_{\mathrm{i}}(\mathrm{l})=\left[\begin{array}{ccccc}
\Omega_{\mathrm{i}} & \Lambda_{\mathrm{i}} & \Xi_{\mathrm{i}}^{13}(\mathrm{I}) & \varepsilon_{\mathrm{i}} \mathrm{U}_{\mathrm{i}}^{\top} & \Phi_{\mathrm{i}} \\
* & -\gamma \mid-\mathrm{H}_{\mathrm{i}} & 0 & 0 & 0 \\
* & * & -\mathrm{R}_{2} & 0 & 0 \\
* & * & * & -\varepsilon_{\mathrm{i}} \mid & \varepsilon_{\mathrm{i}} J^{\top} \\
* & * & * & * & -\varepsilon_{\mathrm{i}} \mathrm{I}
\end{array}\right]<0,
$$

(8)

where

$$
\begin{aligned}
& \Omega_{\mathrm{i}}=\left[\begin{array}{cccc}
-\mathrm{R}_{1}+\sum_{\mathrm{i}=1}^{3} \mathrm{Q}_{1} & \mathrm{P}+\mathrm{A}_{\mathrm{i}}^{\top} \mathrm{S}_{1}^{\top} & \mathrm{R}_{1} & 0 \\
* & \Omega_{\mathrm{i}}^{22} & 0 & \Omega_{\mathrm{i}}^{24} \\
* & * & \Omega_{\mathrm{i}}^{33} & \Omega_{\mathrm{i}}^{34} \\
* & * & * & \Omega_{\mathrm{i}}^{44} \\
* & * & * & * \\
* & * & * & *
\end{array}\right.
\end{aligned}
$$

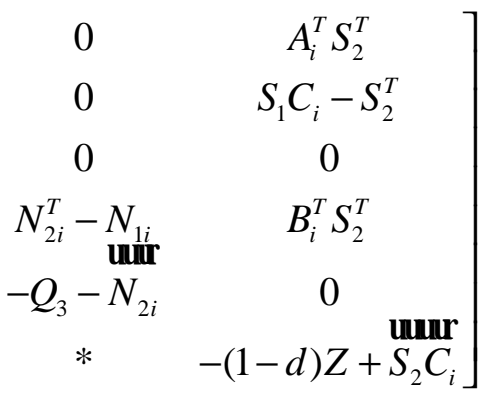

$$
\begin{aligned}
& \Lambda_{\mathrm{i}}^{\top}=\left[-\mathrm{E}_{\mathrm{i}}, \mathrm{D}_{\mathrm{i}}^{\top} \mathrm{S}_{1}^{\top}, 0,-\mathrm{F}_{\mathrm{i}}, 0, \mathrm{D}_{\mathrm{i}}^{\top} \mathrm{S}_{2}^{\top}\right] \text {, } \\
& \Xi_{\mathrm{i}}^{13}(1)=\sqrt{\tau_{\mathrm{M}}-\tau_{\mathrm{m}}} \mathrm{M}_{\mathrm{i}}, \Xi_{\mathrm{i}}^{13}(2)=\sqrt{\tau_{\mathrm{M}}-\tau_{\mathrm{m}}} \mathrm{N}_{\mathrm{i}} \text {, } \\
& \mathrm{E}_{\mathrm{i}}=\left[\mathrm{E}_{1 \mathrm{i}}, 0,0, \mathrm{E}_{2 \mathrm{i}}, 0, \mathrm{E}_{3 \mathrm{i}}\right] \text {, } \\
& \Phi_{1}^{\top}=\left[0, \mathrm{~L}_{\mathrm{i}}^{\top} \mathrm{S}_{1}^{\top}, 0,0,0, \mathrm{~L}_{\mathrm{i}}^{\top} \mathrm{S}_{2}^{\top}\right] \text {, }
\end{aligned}
$$

with

$$
\begin{aligned}
& M_{i}^{\top}=\left[0,0, M_{1 i}^{\top}, M_{2 i}^{\top}, 0,0\right], \\
& N_{i}^{\top}=\left[0,0,0, N_{1 i}^{\top}, N_{2 i}^{\top}, 0\right], \\
& \Omega_{i}^{22}=-S_{1}+\left[\tau_{m} R_{1}+\left(\tau_{M}-\tau_{m}\right) R_{2}+Z\right], \\
& \Omega_{i}^{24}=S_{1} B_{i},
\end{aligned}
$$

$$
\begin{aligned}
& \Omega_{\mathrm{i}}^{33}=-Q_{1}-R_{1}+M_{1 \mathrm{i}}^{\mathbf{m}}, \Omega_{\mathrm{i}}^{34}=M_{2 \mathrm{i}}^{\top}-M_{1 \mathrm{i}}, \\
& \Omega_{\mathrm{i}}^{44}=-(1-\mathrm{d}) Q_{2}+\mathrm{N}_{1 \mathrm{i}}-M_{2 \mathrm{i}} .
\end{aligned}
$$

Proof. Choose the Lyapunov-Krasovskii functional as follows:

$$
\mathrm{V}\left(\mathrm{X}_{\mathrm{t}}\right)=\mathrm{V}_{1}\left(\mathrm{X}_{\mathrm{t}}\right)+\mathrm{V}_{2}\left(\mathrm{x}_{\mathrm{t}}\right)+\mathrm{V}_{3}\left(\mathrm{x}_{\mathrm{t}}\right)+\mathrm{V}_{4}\left(\mathrm{X}_{\mathrm{t}}\right)
$$

where

$$
\begin{gathered}
\mathrm{V}_{1}\left(\mathrm{x}_{\mathrm{t}}\right)=\mathrm{x}^{\top}(\mathrm{t}) \mathrm{P} \mathrm{x}(\mathrm{t}), \\
\mathrm{V}_{2}\left(\mathrm{x}_{\mathrm{t}}\right)=\int_{\mathrm{t}-\tau_{\mathrm{m}}}^{\mathrm{t}} \mathrm{x}^{\top}(\mathrm{s}) \mathrm{Q}_{1} \mathrm{x}(\mathrm{s}) \mathrm{ds}+\int_{\mathrm{t}-\tau(\mathrm{t})}^{\mathrm{t}} \mathrm{x}^{\top}(\mathrm{s}) \mathrm{Q}_{2} \mathrm{x}(\mathrm{s}) \mathrm{ds} \\
+\int_{\mathrm{t}-\tau_{\mathrm{m}}}^{\mathrm{t}} \mathrm{x}^{\top}(\mathrm{s}) \mathrm{Q}_{3} \mathrm{x}(\mathrm{s}) \mathrm{ds}, \\
\mathrm{V}_{3}\left(\mathrm{x}_{\mathrm{t}}\right)=\tau_{\mathrm{m}} \int_{\mathrm{t}-\tau_{\mathrm{m}}}^{\mathrm{t}} \int_{\mathrm{s}}^{\mathrm{t}} \xi^{\top}(\theta) \mathrm{R}_{1} \xi(\theta) \mathrm{d} \theta \mathrm{ds} \\
\quad+\int_{\mathrm{t}-\tau_{\mathrm{m}}}^{\mathrm{t}-\tau_{\mathrm{m}}} \int_{\mathrm{s}}^{\mathrm{t}} \xi^{\top}(\theta) \mathrm{R}_{2} \xi(\theta) \mathrm{d} \theta \mathrm{ds},
\end{gathered}
$$

$$
\mathrm{V}_{4}\left(\mathrm{X}_{\mathrm{t}}\right)=\int_{\mathrm{t}-\tau(\mathrm{t})}^{\mathrm{t}} \xi^{\top}(\mathrm{s}) \mathrm{Z} \xi(\mathrm{s}) \mathrm{ds}
$$

with $\xi(\mathrm{t})=(\mathrm{t})$.

Taking derivative of $\mathrm{V}\left(\mathrm{X}_{\mathrm{t}}\right)$ along the trajectory of the system (6), we have

$$
\begin{aligned}
& V \delta\left(x_{t}\right)=V \delta_{1}\left(x_{t}\right)+V \delta_{2}\left(x_{t}\right)+V \delta_{3}\left(x_{t}\right)+V \delta_{4}\left(x_{t}\right), \\
& \text { where } \\
& V_{1} \mathcal{S}\left(x_{t}\right)=2 x^{\top}(t) P \xi(t) \\
& \mathcal{V}_{2}\left(x_{t}\right)=x^{\top}(t)\left(\sum_{l=1}^{3} Q_{l}\right) x(t)-x^{\top}\left(t-\tau_{m}\right) Q_{1} x\left(t-\tau_{m}\right) \\
& -(1-\tau(\mathrm{t})) \mathrm{x}^{\top}(\mathrm{t}-\tau(\mathrm{t})) \mathrm{Q}_{2} \mathrm{x}(\mathrm{t}-\tau(\mathrm{t})) \\
& -\mathrm{x}^{\top}\left(\mathrm{t}-\tau_{\mathrm{M}}\right) \mathrm{Q}_{3} \mathrm{x}\left(\mathrm{t}-\tau_{\mathrm{M}}\right) \\
& \leq x^{\top}(t)\left(\sum_{l=1}^{3} Q_{1}\right) x(t)-x^{\top}\left(t-\tau_{m}\right) Q_{1} x\left(t-\tau_{m}\right) \\
& -(1-\mathrm{d}) \mathrm{x}^{\top}(\mathrm{t}-\tau(\mathrm{t})) \mathrm{Q}_{2} \mathrm{x}(\mathrm{t}-\tau(\mathrm{t})) \\
& -\mathrm{x}^{\top}\left(\mathrm{t}-\tau_{\mathrm{M}}\right) \mathrm{Q}_{3} \mathrm{x}\left(\mathrm{t}-\tau_{\mathrm{M}}\right) \text {, } \\
& \mathcal{V}_{3}\left(\mathrm{X}_{\mathrm{t}}\right)=\xi^{\top}(\mathrm{t})\left[\tau_{\mathrm{m}} \mathrm{R}_{1}+\left(\tau_{\mathrm{M}}-\tau_{\mathrm{m}}\right) \mathrm{R}_{2}\right] \xi(\mathrm{t}) \\
& -\tau_{\mathrm{m}} \int_{\mathrm{t}-\tau_{\mathrm{m}}}^{\mathrm{t}} \xi^{\top}(\mathrm{s}) \mathrm{R}_{1} \xi(\mathrm{s}) \mathrm{ds} \\
& -\int_{\mathrm{t}-\tau_{\mathrm{M}}}^{\mathrm{t}-\tau_{\mathrm{m}}} \xi^{\top}(\mathrm{s}) \mathrm{R}_{2} \xi(\mathrm{s}) \mathrm{ds}
\end{aligned}
$$

$\bigvee_{4}\left(\mathrm{x}_{\mathrm{t}}\right) \leq \xi^{\top}(\mathrm{t}) \mathrm{Z} \xi(\mathrm{t})-(1-\mathrm{d}) \xi^{\top}(\mathrm{t}-\tau(\mathrm{t})) \mathrm{Z} \xi(\mathrm{t}-\tau(\mathrm{t}))$.

Employing the free-weighing matrix method [19-21], we have

$2 \sum_{\mathrm{i}=1}^{\mathrm{r}} \mu_{\mathrm{i}} \varsigma^{\top}(\mathrm{t}) \mathrm{M}_{\mathrm{i}}\left[\mathrm{x}\left(\mathrm{t}-\tau_{\mathrm{m}}\right)-\mathrm{x}(\mathrm{t}-\tau(\mathrm{t}))-\int_{\mathrm{t}-\tau(\mathrm{t})}^{\mathrm{t}-\tau_{\mathrm{m}}} \xi(\mathrm{s}) \mathrm{ds}\right]=0$, 


$$
\begin{gathered}
2 \sum_{\mathrm{i}=1}^{\mathrm{r}} \mu_{\mathrm{i}} \varsigma^{\top}(\mathrm{t}) \mathrm{N}_{\mathrm{i}}\left[\mathrm{x}(\mathrm{t}-\tau(\mathrm{t}))-\mathrm{x}\left(\mathrm{t}-\tau_{\mathrm{M}}\right)-\int_{\mathrm{t}-\tau_{\mathrm{M}}}^{\mathrm{t}-\tau(\mathrm{t})} \xi(\mathrm{s}) \mathrm{d} \mathrm{s}\right]=0, \\
2 \sum_{\mathrm{i}=1}^{\mathrm{r}} \mu_{\mathrm{i}} \varsigma^{\top}(\mathrm{t}) S\left[-\xi(\mathrm{t})+\mathrm{A}_{\mathrm{i}}(\mathrm{t}) \mathrm{x}(\mathrm{t})+\mathrm{B}_{\mathrm{i}}(\mathrm{t}) \mathrm{x}(\mathrm{t}-\tau(\mathrm{t}))\right. \\
\left.+\mathrm{C}_{\mathrm{i}}(\mathrm{t})(\mathrm{t}-\tau(\mathrm{t}))+\mathrm{D}_{\mathrm{i}} \mathrm{w}(\mathrm{t})\right]=0,
\end{gathered}
$$

where

$$
\begin{aligned}
& \varsigma^{\top}(\mathrm{t})=\left[\mathrm{x}^{\top}(\mathrm{t}), \xi^{\top}(\mathrm{t}), \mathrm{x}^{\top}\left(\mathrm{t}-\tau_{\mathrm{m}}\right), \mathrm{x}^{\top}(\mathrm{t}-\tau(\mathrm{t})), \mathrm{x}^{\top}\left(\mathrm{t}-\tau_{\mathrm{M}}\right),\right. \\
& \left.\xi^{\top}(\mathrm{t}-\tau(\mathrm{t}))\right] \text { and } \mathrm{S}^{\top}=\left[0, \mathrm{~S}_{1}^{\top}, 0,0,0, \mathrm{~S}_{2}^{\top}\right] .
\end{aligned}
$$

Then it follows from (10)-(17) that

$$
\begin{aligned}
& v \&\left(x_{t}\right)-2 w^{\top}(t) y(t)-\gamma w^{\top}(t) w(t) \\
& \leq 2 \mathrm{x}^{\top}(\mathrm{t}) \mathrm{P} \xi(\mathrm{t})+\mathrm{x}^{\top}(\mathrm{t})\left(\sum_{\mathrm{l}=1}^{3} \mathrm{Q}_{\mathrm{I}}\right) \mathrm{x}(\mathrm{t}) \\
& -\mathrm{x}^{\top}\left(\mathrm{t}-\tau_{\mathrm{m}}\right) \mathrm{Q}_{1} \mathrm{x}\left(\mathrm{t}-\tau_{\mathrm{m}}\right) \\
& -(1-\mathrm{d}) \mathrm{x}^{\top}(\mathrm{t}-\tau(\mathrm{t})) \mathrm{Q}_{2} \mathrm{x}(\mathrm{t}-\tau(\mathrm{t})) \\
& -\mathrm{X}^{\top}\left(\mathrm{t}-\tau_{\mathrm{M}}\right) \mathrm{Q}_{3} \mathrm{x}\left(\mathrm{t}-\tau_{\mathrm{M}}\right) \\
& +\xi^{\top}(\mathrm{t})\left[\tau_{\mathrm{m}} \mathrm{R}_{1}+\left(\tau_{\mathrm{M}}-\tau_{\mathrm{m}}\right) \mathrm{R}_{2}+\mathrm{Z}\right] \xi(\mathrm{t}) \\
& -\tau_{\mathrm{m}} \int_{\mathrm{t}-\tau_{\mathrm{m}}}^{\mathrm{t}} \xi^{\top}(\mathrm{s}) \mathrm{R}_{1} \xi(\mathrm{s}) \mathrm{ds} \\
& -\int_{\mathrm{t}-\tau_{\mathrm{M}}}^{\mathrm{t}-\tau_{\mathrm{m}}} \xi^{\top}(\mathrm{s}) \mathrm{R}_{2} \xi(\mathrm{s}) \mathrm{ds} \\
& -(1-\mathrm{d}) \xi^{\top}(\mathrm{t}-\tau(\mathrm{t})) \mathrm{Z} \xi(\mathrm{t}-\tau(\mathrm{t})) \\
& +2 \sum_{\mathrm{i}=1}^{\mathrm{r}} \mu_{\mathrm{i}} \varsigma^{\top}(\mathrm{t}) \mathrm{M}_{\mathrm{i}}\left[\mathrm{x}\left(\mathrm{t}-\tau_{\mathrm{m}}\right)-\mathrm{x}(\mathrm{t}-\tau(\mathrm{t}))\right. \\
& \left.-\int_{\mathrm{t}-\tau(\mathrm{t})}^{\mathrm{t}-\tau_{\mathrm{m}}} \xi(\mathrm{s}) \mathrm{ds}\right] \\
& +2 \sum_{\mathrm{i}=1}^{\mathrm{r}} \mu_{\mathrm{i}} \varsigma^{\top}(\mathrm{t}) \mathrm{N}_{\mathrm{i}} \mathrm{x}(\mathrm{t}-\tau(\mathrm{t}))-\mathrm{x}\left(\mathrm{t}-\tau_{\mathrm{M}}\right) \\
& \left.-\int_{\mathrm{t}-\tau_{\mathrm{M}}}^{\mathrm{t}-\tau(\mathrm{t})} \xi(\mathrm{s}) \mathrm{ds}\right] \\
& +2 \sum_{\mathrm{i}=1}^{\mathrm{r}} \mu_{\mathrm{i}} \varsigma^{\top}(\mathrm{t}) \mathrm{S}\left[-\xi(\mathrm{t})+\mathrm{A}_{\mathrm{i}}(\mathrm{t}) \mathrm{x}(\mathrm{t})\right. \\
& +\mathrm{B}_{\mathrm{i}}(\mathrm{t}) \mathrm{x}(\mathrm{t}-\tau(\mathrm{t})) \\
& \left.+\mathrm{C}_{\mathrm{i}}(\mathrm{t}) \&(\mathrm{t}-\tau(\mathrm{t}))+\mathrm{D}_{\mathrm{i}} \mathrm{w}(\mathrm{t})\right] \\
& -2 \mathrm{w}^{\top}(\mathrm{t}) \mathrm{y}(\mathrm{t})-\gamma \mathrm{w}^{\top}(\mathrm{t}) \mathrm{w}(\mathrm{t}) \text {. }
\end{aligned}
$$

Using Lemma 1 in [10], we have

$$
-\tau_{\mathrm{m}} \int_{\mathrm{t}-\tau_{\mathrm{m}}}^{\mathrm{t}} \xi^{\top}(\mathrm{s}) \mathrm{R}_{1} \xi(\mathrm{s}) \mathrm{ds}
$$$$
\leq\left[\begin{array}{c}
x(t) \\
x\left(t-\tau_{m}\right)
\end{array}\right]^{\top}\left[\begin{array}{cc}
-R_{1} & R_{1} \\
R_{1} & -R_{1}
\end{array}\right]\left[\begin{array}{c}
x(t) \\
x\left(t-\tau_{m}\right)
\end{array}\right] .
$$

Via the method in [7], we obtain

$$
\begin{aligned}
& -2 \sum_{\mathrm{i}=1}^{\mathrm{r}} \mu_{\mathrm{i}} \varsigma^{\top}(\mathrm{t}) \mathrm{M}_{\mathrm{i}} \int_{\mathrm{t}-\tau(\mathrm{t})}^{\mathrm{t}-\tau_{\mathrm{m}}} \xi(\mathrm{s}) \mathrm{d} \mathrm{s} \\
& \leq \int_{\mathrm{t}-\tau(\mathrm{t})}^{\mathrm{t}-\tau_{\mathrm{m}}} \xi^{\top}(\mathrm{s}) \mathrm{R}_{2} \xi(\mathrm{s}) \mathrm{d} s \\
& +\left(\tau(\mathrm{t})-\tau_{\mathrm{m}}\right) \sum_{\mathrm{i}=1}^{\mathrm{r}} \mu_{\mathrm{i}} \varsigma^{\top}(\mathrm{t}) \mathrm{M}_{\mathrm{i}} \mathrm{R}_{2}^{-1} \mathrm{M}_{\mathrm{i}}^{\top} \varsigma(\mathrm{t}),
\end{aligned}
$$

and

$$
\begin{aligned}
& -2 \sum_{\mathrm{i}=1}^{\mathrm{r}} \mu_{\mathrm{i}} \varsigma^{\top}(\mathrm{t}) \mathrm{N}_{\mathrm{i}} \int_{\mathrm{t}-\tau_{\mathrm{M}}}^{\mathrm{t}-\tau(\mathrm{t})} \xi(\mathrm{s}) \mathrm{d} \mathrm{s} \\
& \leq \int_{\mathrm{t}-\tau_{\mathrm{M}}}^{\mathrm{t}-\tau(\mathrm{t})} \xi^{\top}(\mathrm{s}) \mathrm{R}_{2} \xi(\mathrm{s}) \mathrm{d} \mathrm{s} \\
& \quad+\left(\tau_{\mathrm{M}}-\tau(\mathrm{t})\right) \sum_{\mathrm{i}=1}^{\mathrm{r}} \mu_{\mathrm{i}} \varsigma^{\top}(\mathrm{t}) \mathrm{N}_{\mathrm{i}} \mathrm{R}_{2}^{-1} \mathrm{~N}_{\mathrm{i}}^{\top} \varsigma(\mathrm{t}) .
\end{aligned}
$$

So it follows from (18)-(21) that

$$
\begin{aligned}
& v \mathcal{Q}\left(x_{\mathrm{t}}\right)-2 \mathrm{w}^{\top}(\mathrm{t}) \mathrm{y}(\mathrm{t})-\gamma \mathrm{w}^{\top}(\mathrm{t}) \mathrm{w}(\mathrm{t}) \\
& \leq \sum_{\mathrm{i}=1}^{\mathrm{r}} \mu_{\mathrm{i}} \varsigma^{\top}(\mathrm{t})\left[\left(\tau(\mathrm{t})-\tau_{\mathrm{m}}\right) \mathrm{M}_{\mathrm{i}} \mathrm{R}_{2}^{-1} \mathrm{M}_{\mathrm{i}}^{\top}\right. \\
& \left.+\left(\tau_{\mathrm{M}}-\tau(\mathrm{t})\right) \mathrm{N}_{\mathrm{i}} \mathrm{R}_{2}^{-1} \mathrm{~N}_{\mathrm{i}}^{\top}+\Omega_{\mathrm{i}}(\mathrm{t})\right] \varsigma(\mathrm{t}) \\
& +2 \sum_{\mathrm{i}=1}^{\mathrm{r}} \mu_{\mathrm{i}} \varsigma^{\top}(\mathrm{t}) \mathrm{S} \mathrm{D}_{\mathrm{i}} \mathrm{w}(\mathrm{t}) \\
& -2 \mathrm{w}^{\top}(\mathrm{t}) \mathrm{y}(\mathrm{t})-\gamma \mathrm{w}^{\top}(\mathrm{t}) \mathrm{w}(\mathrm{t}) \\
& =\sum_{\mathrm{i}=1}^{\mathrm{r}} \mu_{\mathrm{i}}\left[\begin{array}{c}
\varsigma(\mathrm{t}) \\
\mathrm{w}(\mathrm{t})
\end{array}\right]^{\top}\left[\begin{array}{rr}
\Upsilon_{\mathrm{i}}(\mathrm{t}) & \Lambda_{\mathrm{i}} \\
* & -\gamma \mid-\mathrm{H}_{\mathrm{i}}
\end{array}\right]\left[\begin{array}{l}
\varsigma(\mathrm{t}) \\
\mathrm{w}(\mathrm{t})
\end{array}\right]
\end{aligned}
$$

where

$\Upsilon_{i}(\mathrm{t})=\left(\tau(\mathrm{t})-\tau_{\mathrm{m}}\right) \mathrm{M}_{\mathrm{i}} \mathrm{R}_{2}^{-1} \mathrm{M}_{\mathrm{i}}^{\top}+\left(\tau_{\mathrm{M}}-\tau(\mathrm{t})\right) \mathrm{N}_{\mathrm{i}} \mathrm{R}_{2}^{-1} \mathrm{~N}_{\mathrm{i}}^{\top}+\Omega_{\mathrm{i}}(\mathrm{t})$ and $\Omega_{\mathrm{i}}(\mathrm{t})$ is obtained form $\Omega_{\mathrm{i}}$ by replacing the terms $A_{i}, B_{i}, C_{i}$ with $A_{i}(t), B_{i}(t), C_{i}(t)$, respectively.

By the Schur complements, Lemma 1 and Lemma 2, the LMIs (8) give that

$$
\left[\begin{array}{cr}
\Upsilon_{\mathrm{i}}(\mathrm{t}) & \Lambda_{\mathrm{i}} \\
* & -\gamma \mid-\mathrm{H}_{\mathrm{i}}
\end{array}\right]<0, \mathrm{i} \in \mathrm{S} .
$$

Then, it follows from (22) and (23) that

$$
V \mathcal{S}\left(x_{t}\right)-2 w^{\top}(t) y(t)-\gamma w^{\top}(t) w(t)<0 \text {. }
$$
Integrating (24) with respect to $t$ over time interval $\left[0, \mathrm{t}_{\rho}\right], \mathrm{t}_{\rho} \geq 0$, we have

$$
\begin{aligned}
\mathrm{V}\left(\mathrm{x}_{\mathrm{t}_{\rho}}\right) & -\mathrm{V}\left(\mathrm{x}_{0}\right)-\gamma \int_{0}^{\mathrm{t}_{\rho}} \mathrm{w}^{\top}(\mathrm{s}) \mathrm{w}(\mathrm{s}) \mathrm{ds} \\
& \leq 2 \int_{0}^{\mathrm{t}_{\rho}} \mathrm{w}^{\top}(\mathrm{s}) \mathrm{y}(\mathrm{s}) \mathrm{ds} .
\end{aligned}
$$

So, under the zero initial condition, we have $-\gamma \int_{0}^{t_{\rho}} \mathrm{w}^{\top}(\mathrm{s}) \mathrm{w}(\mathrm{s}) \mathrm{d} \mathrm{s} \leq 2 \int_{0}^{\mathrm{t}_{\rho}} \mathrm{w}^{\top}(\mathrm{s}) \mathrm{y}(\mathrm{s}) \mathrm{d} \mathrm{s}$.

The proof is completed here.

Remark 2. Letting $\mathbf{J}=0$ in (8) yields the Theorem 1 in [22]. In view of this, our results in the article extend the corresponding results in [22]. 


\section{SIMULATION EXAMPLE}

In this section, a simulation example is given to illustrate the effectiveness of the developed approach. Consider the system (1) with parameters as follows

$$
\begin{aligned}
& A_{1}=\left[\begin{array}{cc}
-4 & 0 \\
1.2 & -5
\end{array}\right], \quad A_{2}=\left[\begin{array}{cc}
-3 & 0.6 \\
0 & -4
\end{array}\right] \text {, } \\
& B_{1}=\left[\begin{array}{cc}
1 & 0.4 \\
0.5 & 1.3
\end{array}\right], \quad B_{2}=\left[\begin{array}{cc}
14 & 0.6 \\
0.4 & 0.8
\end{array}\right] \text {, } \\
& C_{1}=\left[\begin{array}{cc}
0.4 & 0 \\
0 & -0.2
\end{array}\right], \quad C_{2}=\left[\begin{array}{cc}
-0.2 & 0 \\
0 & 0.3
\end{array}\right] \text {, } \\
& D_{1}=\left[\begin{array}{cc}
1.2 & -0.4 \\
0.6 & 1.5
\end{array}\right], \quad D_{2}=\left[\begin{array}{cc}
-0.9 & -0.8 \\
0.3 & 1
\end{array}\right] \text {, } \\
& E_{1}=\left[\begin{array}{ll}
-1.6 & 0.8 \\
-0.2 & 1.3
\end{array}\right], \quad E_{2}=\left[\begin{array}{cc}
-14 & -0.2 \\
0.6 & 1.5
\end{array}\right] \text {, } \\
& F_{1}=\left[\begin{array}{cc}
0.8 & 0.6 \\
-0.5 & -1
\end{array}\right], \quad F_{2}=\left[\begin{array}{cc}
-1 & 0.3 \\
0 & 0.6
\end{array}\right] \text {, } \\
& H_{1}=\left[\begin{array}{cc}
-0.6 & -0.5 \\
0.4 & 1
\end{array}\right], H_{2}=\left[\begin{array}{cc}
12 & 0.6 \\
0.4 & -0.5
\end{array}\right] \text {, } \\
& L_{1}=\left[\begin{array}{cc}
1 & -0.4 \\
0.6 & 1.2
\end{array}\right], \quad L_{2}=\left[\begin{array}{cc}
0.6 & 0 \\
0.2 & 0.8
\end{array}\right] \text {, } \\
& E_{11}=\left[\begin{array}{cc}
0.6 & -0.3 \\
0.2 & 0.7
\end{array}\right], \quad E_{12}=\left[\begin{array}{cc}
1.2 & 0 \\
0.4 & -0.5
\end{array}\right] \text {, } \\
& E_{21}=\left[\begin{array}{cc}
-0.8 & 0 \\
0.5 & -1.2
\end{array}\right], E_{22}=\left[\begin{array}{cc}
-1 & 0.3 \\
0 & 0.45
\end{array}\right] \text {, } \\
& E_{31}=\left[\begin{array}{cc}
1.2 & -0.6 \\
0 & -1
\end{array}\right], \quad E_{32}=\left[\begin{array}{cc}
0.8 & 0.2 \\
0.4 & -11
\end{array}\right] \text {, } \\
& J=\left[\begin{array}{cc}
0.2 & 0 \\
0 & 0.1
\end{array}\right], \quad F(t)=\left[\begin{array}{cc}
\sin t & 0 \\
0 & \cos t
\end{array}\right], \\
& \tau(\mathrm{t})=1-2 \sin \mathrm{t} .
\end{aligned}
$$

Choose the scalar $\gamma=1.25$, then solving the LMIs in (8) via the algorithm "feasp" in Matlab, it is found that these LMIs are feasible. So, according to Theorem 1, the system (1) is passive. For convenience of the simulation, let

$$
\mu_{1}(\mathrm{t})=\sin \left(\pi \mathrm{x}_{1}(\mathrm{t})\right), \mu_{2}(\mathrm{t})=1-\sin \left(\pi \mathrm{x}_{1}(\mathrm{t})\right)
$$

and

$$
w(t)=\left[\begin{array}{ll}
\frac{1}{1+t} & \frac{1}{2+0.3 t}
\end{array}\right]^{\top}, t \geq 0 .
$$

Then, the simulation results of the state response of the plant is given in Fig.1, while Fig.2 shows the system output. And the curve of

$$
J\left(\mathrm{t}_{\rho}\right)=\frac{-2 \int_{0}^{\mathrm{t}_{\rho}} \mathrm{w}^{\top}(\mathrm{s}) \mathrm{y}(\mathrm{s}) \mathrm{ds}}{\int_{0}^{\mathrm{t}_{\rho}} \mathrm{w}^{\top}(\mathrm{s}) \mathrm{W}(\mathrm{s}) \mathrm{ds}}, \mathrm{t}_{\rho} \geq 0
$$

is provided in Fig. 3.

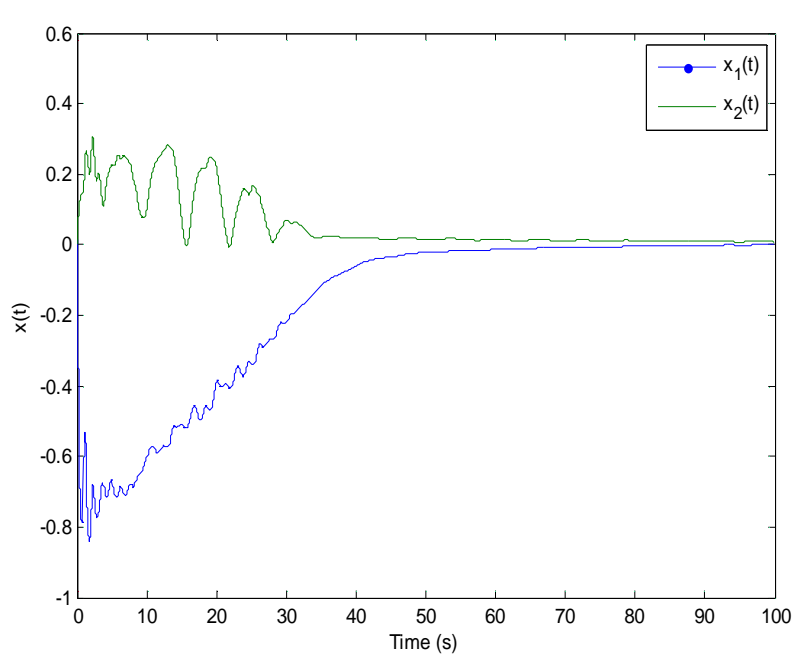

Fig. 1. State response $x(t)$.

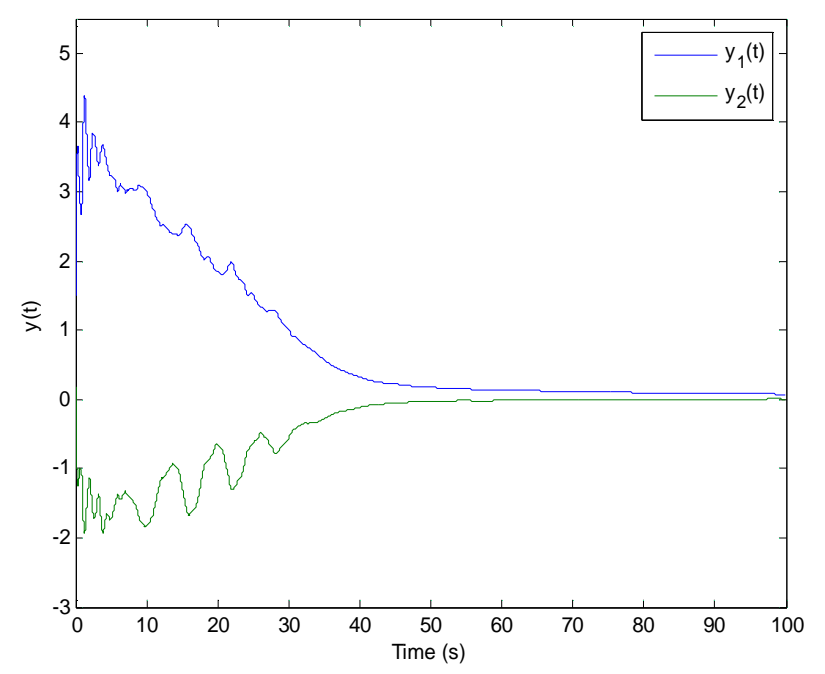

Fig. 2. System output y(t). 


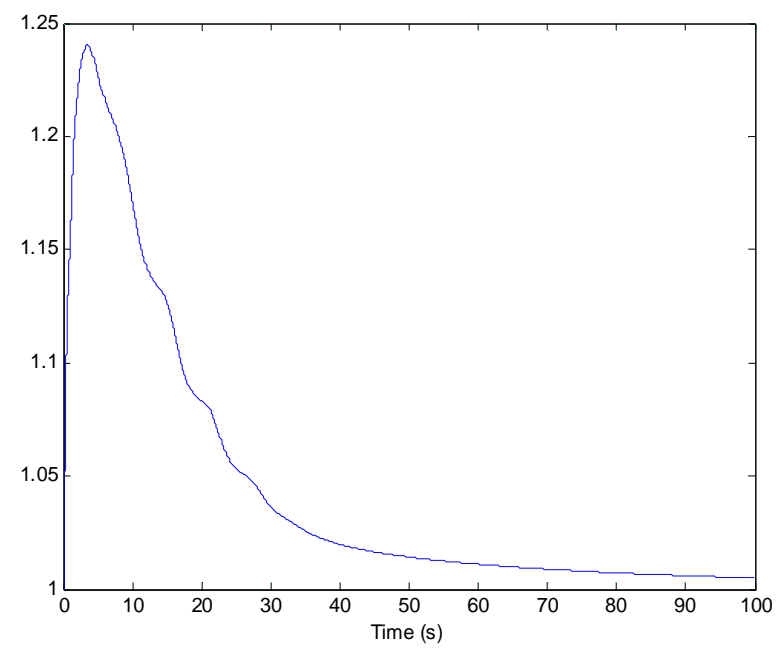

Fig. 3. The curve of $\mathrm{J}\left(\mathrm{t}_{o}\right)$.

\section{CONCLUSION}

This paper has investigated the passivity problem for the T-S fuzzy neutral system with interval time-varying delay and linear fractional parametric uncertainties. Delay dependent sufficient conditions for solvability of the passive problem are obtained by means of the LyapunovKrasovskii functional and the free weighting matrix method. The presented criterion in terms of LMIs can be readily solved via the standard numerical algorithm in Matlab. Finally, a simulation example is provided to demonstrate effectiveness and applicability of the theoretical results.

\section{ACKNOWLEDGMENT}

The authors would like to thank ICIECS2010 and the anonymous reviewers for their helpful and insightful comments for further improving the quality of this work.

\section{REFERENCES}

[1] T. Takagi and M. Sugeno, "Fuzzy identification of systems and its application to modeling and control," IEEE Trans. Syst., Man and Cyb., vol.15, pp.116-132, 1985.

[2] S. Xu, J. Lam, and B. Chen, "Robust $H_{\infty}$ control for uncertain fuzzy neutral delay systems," European J. Contr., vol.10, pp.365-380,2004.

[3] Y. Li and S. Xu, "Robust stabilization and $\mathrm{H}_{\infty}$ control for uncertain fuzzy neutral systems with mixed time delays," Fuzzy Sets and Syst., vol. 159, pp. 2730-2748, 2008.
[4] J. Yang, S. Zhong, and L. Xiong, "A descriptor system approach to non-fragile $H_{\infty}$ control for uncertain fuzzy neutral systems," Fuzzy Sets and Syst., vol. 160, pp. 423438, 2009.

[5] J. Yang, S. Zhong, G. Li, and W. Luo, "Robust $H_{\infty}$ filter design for uncertain fuzzy neutral systems," Inform. Sci., vol. 179, pp. 3697-3710, 2009.

[6] J. Yang, W. Luo, G. Li, and S. Zhong, "Reliable guaranteed cost control for uncertain fuzzy neutral systems," Nonlinear Analysis: Hybrid Systems, vol. 4, pp. 644-658, 2010.

[7] D. Yue, Q. Han, and C. Peng, "State feedback controller design of networked controls ystems," IEEE Trans. Circuits Syst.II, vol. 51, pp. 640-644, 2004.

[8] X. Jiang and Q. Han, "Robust H 1 control for uncertain Takagi-Sugeno fuzzy system with interval time-varying delay," IEEE Trans. Fuzzy Syst., vol. 15, pp. 321-331, 2007.

[9] C. Lien, K. Yu, W. Chen, Z. Wan and Y. Chung, "Stability criteria for uncertain Takagi-Sugeno fuzzy systems with interval time-varying delay," IET Proc. Control Theory Appl., vol. 1, pp. 764-769, 2007.

[10] C. Peng and Y. Tian, "Delay-dependent robust stability criteria for uncertain systems with interval time-varying delay," J. Comput. Appl. Math., vol. 214, pp. 480-494, 2008.

[11] E. Tian, D. Yue, and Y. Zhang, "Delay-dependent robust $\mathrm{H}_{\infty}$ control for T-S fuzzy system with interval timevarying delay," Fuzzy Sets and Systems, vol. 160, pp. 1708-1719, 2009.

[12] D. Hill and P. Moylan, "The stability of nonlinear dissipative systems," IEEE Trans. on Auto. Contr., vol.21, pp.708-711, 1976.

[13] X. Liu, "Passivity analysis of uncertain fuzzy delayed systems," Chaos, Solitons \& Fractals, vol.34, pp. 833-838, 2007.

[14] G. Calcev, R. Gorez, and M. De Neyer, "Passivity approach to fuzzy control systems," Automatica, vol.33, pp. 339-344, 1998.

[15] C. Li, H. Zhang, and X. Liao, "Passivity and passification of uncertain fuzzy systems," IEE Proc.-Circuits Devices Systems, vol.152, pp. 649- 653, 2005.

[16] J. Liang, Z. Wang, and X. Liu, "Robust passivity and passification of stochastic fuzzy time-delay systems," Information Sciences, vol. 180, pp. 1725-1737, 2010.

[17] R. Lozano, B. Brogliato, O. Egeland, and B. Maschke, "Dissipative systems analysis and control: theory and applications," Springer- Verlag, London, 2000.

[18] S. Zhou, G. Feng, J. Lam, S. Xu, Robust $H_{\infty}$ control for discrete-time fuzzy systems via basis-dependent Lyapunov functions, Infor. Sci. 174 (2005) 197-217.

[19] Y.He, M.Wu, J.H.She, G.P.Liu,Delay-dependent robust stability criteria for uncertain neutral systems with mixed delays, Syst. Control Lett. 51 (2004)57-65.

[20] Y.He, M.Wu, J.H.She, G.P.Liu, Parameter-dependent Lyapunov functional for stability of time-delay systems with polytopic-type uncertainties, IEEE Trans. Autom.Contro 149(2004)828-832.

[21] D.Yue, Q.-L.Han, Delay-dependent exponential stability of stochastic systems with time-varying delay, nonlinearity 
and Markovian switching, IEEE Trans. Autom. Control 50(2005)217-222.

[22] Wenpin Luo and Jun Yang, "Passivity analysis of uncertain neutral fuzzy system with interval time-varying delay," The 2nd Int. Conf. on Information Engineering and Computer Science, vol. 2, pp. 1002-1005, 2010.

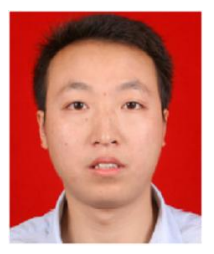

J un Y ang was born in Luzhou, Sichuan province in 1981, and received the B.S. degree from Leshan Normal University, Leshan, China, in 2004 and the Ph.D. degree from University of Electronic Science and Technology of China, Chengdu, China, in 2009, all in Applied Mathematics. His current research interests include fuzzy control systems, stochastic systems and hybrid dynamic systems.

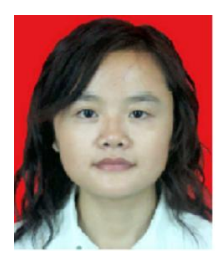

Wenpin Luo was born in Chengdu, Sichuan province in 1981, and received the B.S. degree from Sichuan Normal University, Chengdu, China, in 2004 and the M.S. degree from University of Electronic Science and Technology of China, Chengdu, China, in 2007, all in Applied Mathematics. Her current research interests

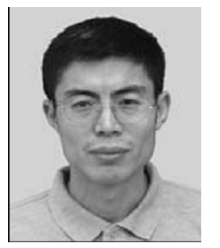

Jinzhong Cui was born in 1966 in Shanxi, China. He received his B.S. and M.S. degrees from the School of Computer Science and Engineering, University of Electronic Science and Technology of China (UESTC) in 1989 and 1994, respectively. Now he is an associate professor with UESTC. His research interests include control theory, Computer Control Techno- logy and Embedded System. include fuzzy control systems, impulsive systems and neural networks. 